\title{
ESTUDIO BIBLIOMÉTRICO DE LA REVISTA PSICOONCOLOGÍA (2003-2015)
}

\author{
BIBLIOMETRIC STUDY OF THE JOURNAL PSICOONCOLOGÍA (2003-2015)
}

\author{
Diego Morena de Diago' y Beatriz Morena de Diago² \\ I Servicio de Atención Rural de Villaviciosa de Odón \\ 2 Universidad Complutense de Madrid
}

\section{Resumen}

A través de este estudio bibliométrico se pretende mostrar cómo ha sido la trayectoria de la revista en los 12 años de vida con que cuenta. Se muestra cuál ha sido la producción por años, por autor, por tipología de autor (personal y corporativo), por género, por la procedencia geográfica de los autores (países y comunidades autónomas), por afiliación institucional de los mismos, por el número de firmantes de los artículos y, por último, analizar también la temática a través de las palabras clave propuestas por los, autores de los trabajos.

Palabras clave: Análisis Bibliométrico, Revista, Indicador Bibliométrico; Psicooncología, Cáncer.

\section{Abstract}

This bibliometric study seeks to present the trajectory of the journal through its 12 years of life. The evaluation shows the production of articles by the number of papers per year, by author, author typology (individual or collective), sex, geographic origin of the authors (countries or autonomous regions), institutional affiliation of the authors, number of signatures in the different articles and, finally, analyze the subjects using the different keywords given by the authors.

Keywords: Bibliometric Analysis; Journal, Bibliometric Indicator, Psycho-Oncology, Cancer.

\section{INTRODUCCIÓN}

La Revista de Psicooncología: Investigación y Clínica Biopsicosocial en Oncología fue fundada en el año 2003 por Juan Antonio Cruzado como vehículo de difusión de trabajos teóricos, experimentales y clínicos sobre aspectos psicológicos, sociales, éticos y de calidad de vida asociados al cáncer. Según los datos recogi- dos en el Instituto Nacional de Estadística (INE) el cáncer es la segunda causa de muerte en España. Por sexo, los tumores fueron la primera causa de muerte en los hombres (con una tasa de 296,3 fallecidos por cada 100.000) y la segunda en mujeres (con 180,0 por cada 100.000).

La revista se publica por el Servicio de Publicaciones de la Universidad Complutense de Madrid con una frecuencia de

\section{Correspondencia:}

Diego Morena de Diago

Centro de Salud de Navalcarnero

Calle de la doctora 10 - 28600, Navalcarnero (Madrid)

E-mail: moreago@gmail.com 
publicación cuatrimestral y es indexada en SCOPUS, IBECS, IME, CINDOC, Latindex, PSICODOC, Dialnet, IN-RECS, etc.

La Revista está enmarcada dentro del área de las Ciencias de la Salud, estando la sede de esta publicación en la Facultad de Psicología de la Universidad Complutense de Madrid.

La estructura interna de la revista en su número 2-3 (2015) responde a la dirección de Juan Antonio Cruzado, tres secretarias, Helena Olivera Pérez-Frade, María Soriano Cirugeda y Ana Sanz Cortés y un consejo de redacción formado, en su mayor parte, por profesionales con afiliaciones a universidades nacionales.

Cada artículo es evaluado por el procedimiento denominado "doble ciego", que consiste en la evaluación de los artículos por dos expertos externos, que no se conocen entre sí ni conocen la identidad del autor o autores del trabajo, consiguiendo así una mayor neutralidad en su evaluación (Burgos Bordonau y Palacios Gómez, p. 85) ${ }^{(1)}$.

\section{RESULTADOS}

\section{Producción por años}

La Revista de Psicooncología ha publicado un total de 376 artículos desde el primer número de 2003 hasta el último en 2015. A partir de los datos representados en la Figura 1 podemos realizar las siguientes observaciones referentes a los años de producción:
- Los años más productivos han sido el 2004 (34 artículos), 2006, 2007 y 2009 (33 artículos).

- La más baja producción la arroja el número correspondiente al año 2003 (15 artículos). No hay que olvidar que en 2003 solo se publicó un volumen con un número, a diferencia del resto de años que se publicaron tres números cada año.

- El resto de los años han tenido un número de contribuciones similar, como los años 2005 y 2008 (30 artículos), 2013 (31 artículos), 2010 y 2014 (28 artículos), 2011, 2012 y 2015 (27 artículos).

Véase la figura 1.

Producción de artículos por autor

En la producción de artículos de la revista han intervenido un total de 901 autores personales siendo los 25 más productivos los recogidos en la Tabla 1 (relacionamos los que han producido 5 o más trabajos). Se han consignado las procedencias institucionales más actuales según el año de publicación de las contribuciones (ver la tabla 1). Como puede observarse, encabezan las primeras posiciones Juan Antonio Cruzado con un total de 26 artículos, María Eugenia Olivares con 12 artículos y Javier Barbero con 10 contribuciones respectivamente.

Figura 1. Número de artículos por año

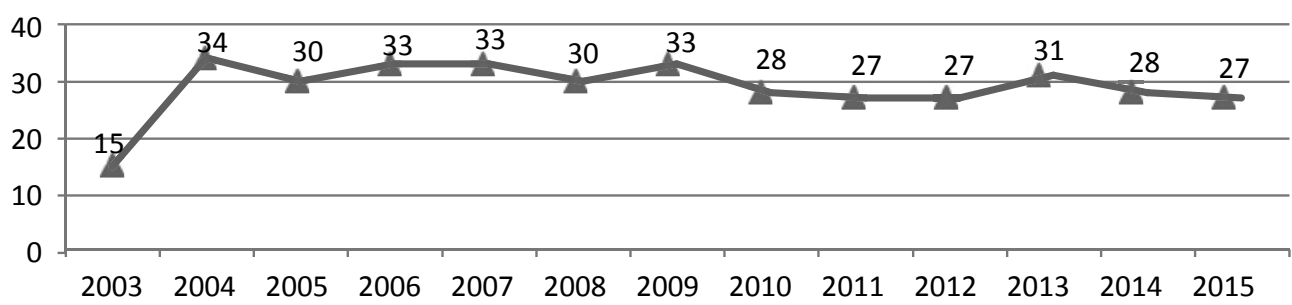


Tabla 1. Filiación de los autores más productivos

\begin{tabular}{|c|c|c|}
\hline Autor & Afiliación & $\begin{array}{l}\text { Número de } \\
\text { artículos }\end{array}$ \\
\hline Adela Cañete & Unidad de Oncología Pediátrica. Hospital La Fe Valencia & 6 \\
\hline Agustina Sirgo Rodríguez & $\begin{array}{l}\text { Departamento de Medicina y Cirugía, Universidad Rovira i } \\
\text { Virgili. Instituto Investigaciones Sanitarias Pere Virgili. Reus. } \\
\text { España }\end{array}$ & 8 \\
\hline Antoni Font & Universitat Autònoma de Barcelona & 9 \\
\hline Belén Fernández & Asociación Española Contra el Cáncer & 8 \\
\hline $\begin{array}{l}\text { Concepción Fernández } \\
\text { Rodríguez }\end{array}$ & Universidad de Oviedo & 5 \\
\hline Eva Rodríguez Bruzos & Universitat Autònoma de Barcelona & 5 \\
\hline Ignacio Blanco & $\begin{array}{c}\text { Unidad de Consejo Genético, Programa de Cáncer Hereditario, } \\
\text { Institut Català d'Oncologia, ICO_IDIBELL }\end{array}$ & 6 \\
\hline Jaime Feliú & $\begin{array}{l}\text { Servicio de Oncología Médica Hospital Universitario La Paz. } \\
\text { IdIPAZ. RETICC RD06/0020/1022. }\end{array}$ & 7 \\
\hline Jaime Sanz & $\begin{array}{c}\text { Servicio de Oncología Médica y Cuidados Paliativos. Hospital } \\
\text { Universitario Marqués de Valdecilla. Santander. } \\
\text { Facultad de Medicina Universidad de Cantabria }\end{array}$ & 6 \\
\hline Javier Barbero & $\begin{array}{l}\text { Servicio Hematología y Hemoterapia Hospital Universitario La } \\
\text { Paz }\end{array}$ & 10 \\
\hline Jordi Bernabeu & $\begin{array}{l}\text { Unidad de Oncología Pediátrica. Hospital La Fe Valencia } \\
\text { Unidad de Daño cerebral Hermanas Hospitalarias. Valencia }\end{array}$ & 5 \\
\hline Jorge Maté & $\begin{array}{l}\text { Unidad de Psico-Oncología Hospital Duran i Reynals. Institut } \\
\text { Català d'Oncologia }\end{array}$ & 6 \\
\hline Juan Antonio Cruzado & Facultad de Psicología, Universidad Complutense de Madrid & 26 \\
\hline Leticia Ascencio Huertas & $\begin{array}{c}\text { Servicio de Cuidados Paliativos del Instituto Nacional de } \\
\text { Cancerología }\end{array}$ & 6 \\
\hline María Eugenia Olivares & $\begin{array}{c}\text { Servicio de Obstetricia y Ginecología. Hospital Clínico San } \\
\text { Carlos. Facultad de Medicina. Universidad Complutense Madrid }\end{array}$ & 12 \\
\hline Manuel González Barón & Hospital Universitario La Paz. Madrid & 5 \\
\hline Manuel Hernández & $\begin{array}{c}\text { Unidad de Psicooncología. Servicio de Psiquiatría. Hospital } \\
\text { Universitario de Basurto. Bilbao. Osakidetza. }\end{array}$ & 5 \\
\hline Óscar Galindo Vázquez & $\begin{array}{l}\text { Servicio de Psico-oncología, Instituto Nacional de Cancerología } \\
\text { INCAN. México }\end{array}$ & 6 \\
\hline Pedro P. Pérez Segura & $\begin{array}{c}\text { Unidad de Consejo genético del Servicio de Oncología Médica } \\
\text { Hospital Clínico San Carlos }\end{array}$ & 9 \\
\hline Pilar Barreto & $\begin{array}{l}\text { Departamento de Personalidad, Evaluación y Tratamientos } \\
\text { Psicológicos, Universidad de Valencia }\end{array}$ & 5 \\
\hline Ramón Bayés & Universitat Autònoma de Barcelona & 7 \\
\hline $\begin{array}{l}\text { Salvador Alvarado } \\
\text { Aguilar }\end{array}$ & Servicio de Psico-oncología, Instituto Nacional de Cancerología. & 6 \\
\hline Sonia López-Santiago & Facultad de Psicología. Universidad Complutense de Madrid & 5 \\
\hline Tina Barahona & $\begin{array}{c}\text { Junta Asociada Provincial de Valencia de la Asociación Española } \\
\text { Contra el Cáncer } \\
\text { Unidad de Oncología Pediátrica. Hospital La Fe Valencia }\end{array}$ & 5 \\
\hline Tomás Blasco & $\begin{array}{c}\text { Departament de Psicologia Bàsica. Universitat Autònoma de } \\
\text { Barcelona }\end{array}$ & 7 \\
\hline
\end{tabular}

Fuente: elaboración propia. 
Producción por género

La suma total de autores asciende a 904, de los cuales 581 son de género femenino y 319 de género masculino. Podemos señalar la escasa contribución en la revista de autores corporativos (ver la tabla 2 y figura 2)

La colaboración científica de los autores

Según el índice de coautoría de los trabajos, partiendo del número de firmantes por artículo, el 21,44\% de los trabajos están firmados por dos autores. La razón bruta autor/trabajo es de 2,42 autores por trabajo y la razón bruta trabajo/autor es de 0,41 trabajos por autor (ver la tabla 3).
Producción por procedencia geográfica

En este apartado analizamos la procedencia geográfica de los autores personales, teniendo en cuenta la afiliación institucional que tenían en el momento de la publicación de sus trabajos.

La mayoría de la producción es de autores españoles, destacando la alta productividad de los autores de la Comunidad de Madrid. En la tabla 4 se ha enumerado la producción por países extranjeros. En primer lugar se posiciona México seguido de Colombia; suponemos que influenciado por el idioma común que facilita la publicación. En la tabla 5 aparecen las publicaciones españolas por comunidades autónomas

\section{Tabla 2. Producción de artículos por género del autor}

\begin{tabular}{|c|c|c|}
\hline Género de los autores & Número de autores & Porcentaje (\%) \\
\hline Mujer & 581 & 64,26 \\
\hline Hombre & 320 & 35,39 \\
\hline Corporativos & 3 & 0,33 \\
\hline Total & 904 & 100 \\
\hline
\end{tabular}

Fuente: elaboración propia.

Figura 2. Distribución de artículos publicados por género de su autor

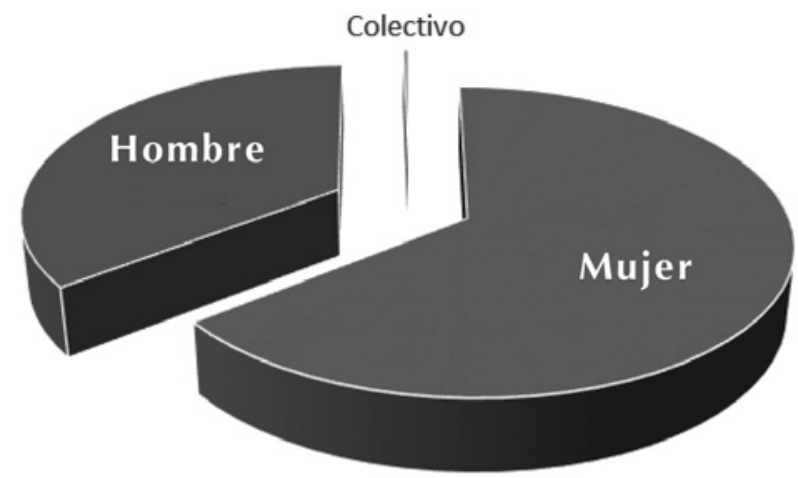

- Mujer - Hombre 


\section{Tabla 3. Colaboración científica entre los autores}

\begin{tabular}{|c|c|c|}
\hline Firmantes por artículo & Número de artículos & Porcentaje (\%) \\
\hline 1 firma & 60 & 16,08 \\
\hline 2 firmas & 80 & 21,44 \\
\hline 3 firmas & 79 & 21,17 \\
\hline 4 firmas & 50 & 13,40 \\
\hline 5 firmas & 32 & 8,57 \\
\hline Más de 5 firmas & 72 & 19,30 \\
\hline Total & 373 & 100 \\
\hline
\end{tabular}

Fuente: elaboración propia.

Tabla 4. Producción geográfica de autores extranjeros

\begin{tabular}{|c|c|}
\hline $\begin{array}{l}\text { Producción por } \\
\text { países extranjeros }\end{array}$ & $\begin{array}{c}\text { Número de } \\
\text { artículos }\end{array}$ \\
\hline Alemania & 1 \\
\hline Argentina & 4 \\
\hline Brasil & 4 \\
\hline Canadá & 3 \\
\hline Chile & 4 \\
\hline Colombia & 12 \\
\hline Dinamarca & 1 \\
\hline Ecuador & 1 \\
\hline Estados Unidos & 17 \\
\hline Italia & 2 \\
\hline Reino Unido & 3 \\
\hline México & 34 \\
\hline Perú & 1 \\
\hline Portugal & 1 \\
\hline Uruguay & 1 \\
\hline Venezuela & 1 \\
\hline Total & 90 \\
\hline
\end{tabular}

Fuente: elaboración propia.
Tabla 5. Producción geográfica de autores en España

\begin{tabular}{|c|c|}
\hline $\begin{array}{l}\text { Producción por } \\
\text { Comunidades } \\
\text { Autónomas }\end{array}$ & $\begin{array}{c}\text { Número de } \\
\text { artículos }\end{array}$ \\
\hline Andalucía & 20 \\
\hline Aragón & 6 \\
\hline Asturias & 5 \\
\hline Baleares & 4 \\
\hline Canarias & 3 \\
\hline Cantabria & 11 \\
\hline Castilla La Mancha & 5 \\
\hline Castilla y León & 7 \\
\hline Cataluña & 72 \\
\hline $\begin{array}{c}\text { Comunidad de } \\
\text { Madrid }\end{array}$ & 137 \\
\hline $\begin{array}{l}\text { Comunidad } \\
\text { Valenciana }\end{array}$ & 43 \\
\hline Extremadura & 4 \\
\hline Galicia & 6 \\
\hline La Rioja & 1 \\
\hline Murcia & 4 \\
\hline Navarra & 6 \\
\hline País Vasco & 6 \\
\hline Total & 340 \\
\hline
\end{tabular}

Fuente: elaboración propia. 


\section{PRODUCCIÓN POR AFILIACIÓN INSTITUCIONAL DE LOS AUTORES}

Las procedencias de las afiliaciones institucionales de los autores de esta revista las hemos reunido en tres grandes apartados.
a. Universidades/Escuelas
b. Hospitales/Centros médicos
c. Asociaciones/Institutos profesionales

La producción por número de firmas ha estado vinculada a 301 instituciones, entre las cuales se han contabilizado un total de 81 universidades/escuelas, 123 hospitales/centros médicos y 97 asociaciones/ institutos profesionales. Muchos autores aparecen en los artículos con más de una afiliación.

El grupo de hospitales y centros médicos es el más numeroso con un total de 665 firmas. Se posiciona en primer lugar el Hospital Universitario La Paz (12,2\%) en segundo lugar el Hospital Clínico San Carlos (10,1\%), ambos de Madrid, en tercer lugar el Hospital Universitario Marqués de Valdecilla (5,9\%) y, en cuarto lugar, el Hospital Universitario La Fe de Valencia (5\%).

El segundo grupo más numeroso es el de las universidades y escuelas. Se han contabilizado un total de 497 firmas concentradas en la Universidad Complutense de Madrid (14,08\%), la Universidad Autónoma de Barcelona (10,46 \%) y la Universidad de Valencia $(7,64 \%)$.

El último grupo, es el más diverso. Recoge un total de 371 firmas repartidas en institutos, fundaciones, agencias, asociaciones, etc. Entre todas ellas, destacamos el Instituto Catalán de Oncología (13,74 $\%$ ), el Instituto Nacional de Cancerología de México (12,39 \%) y la Asociación Española Contra el Cáncer (9,97\%).

En las tablas 6, 7 y 8 pueden verse en detalle la producción por afiliación institucional de los autores contabilizando número de firmas.

\section{Tabla 6. Producción por afiliación institucional de los autores: Universidades/Escuelas}

\begin{tabular}{|c|c|}
\hline Producción por Universidades/Escuelas & $\begin{array}{c}\text { Número } \\
\text { de firmas }\end{array}$ \\
\hline Universidad Complutense de Madrid & 70 \\
\hline Universidad de Gerona & 4 \\
\hline Universidad Autónoma de Barcelona & 52 \\
\hline Universidad de Jaén & 4 \\
\hline Universidad de Valencia & 38 \\
\hline Universidad de Cantabria & 29 \\
\hline Universidad de Santiago de Compostela & 6 \\
\hline Universidad de Murcia & 7 \\
\hline Universidad Miguel Hernández. Elche & 4 \\
\hline Escuela Nacional de Sanidad & 1 \\
\hline Universidad Autónoma de Madrid & 14 \\
\hline Universidad de Oviedo & 17 \\
\hline Universidad de Deusto. Bilbao & 2 \\
\hline Universidad de Almería & 3 \\
\hline Universidad de Granada & 8 \\
\hline Universidad Internacional de Cataluña & 4 \\
\hline Universidad de la Sabana, Bogotá, Colombia & 1 \\
\hline
\end{tabular}

\begin{tabular}{|c|c|}
\hline Universidad de San Luis de Potosí, México & 6 \\
\hline Universidad Jaime I & 12 \\
\hline Universidad de Cádiz & 1 \\
\hline $\begin{array}{c}\text { Cniversidad Pontificia Bolivariana de } \\
\text { Colombia }\end{array}$ & 5 \\
\hline New York University & 3 \\
\hline Universidad Nacional de Educación a \\
Distancia & 4 \\
\hline Universidad Central de Venezuela (UCV) & 6 \\
\hline Universidad de Huelva & 3 \\
\hline Universidad San Pablo-CEU & 2 \\
\hline Universidad de Castilla-La Mancha & 1 \\
\hline Universidad Intercontinental & 1 \\
\hline Universidad de La Laguna. Tenerife & 1 \\
\hline Hospital Universitario Germans Trias y \\
Pujol. Badalona
\end{tabular}




\begin{tabular}{|c|c|}
\hline $\begin{array}{c}\text { Universidade do Rio do Vale dos Sinos } \\
\text { (UNISINOS) }\end{array}$ & 8 \\
\hline Universitat Ramon Llull & 4 \\
\hline $\begin{array}{c}\text { Escola Superior de Música de Catalunya } \\
\text { (ESMUC) }\end{array}$ & 2 \\
\hline Universidad El Bosque. Bogotá & 3 \\
\hline Universidad de Córdoba & 3 \\
\hline Universidad de Zaragoza & 2 \\
\hline Universidad de Buenos Aires, Argentina & 1 \\
\hline Pontificia Universidad Católica de Chile & 5 \\
\hline Universidad Rovira i Virgili & 5 \\
\hline Universidad Nacional de Colombia & 2 \\
\hline Universidad Autónoma de Nuevo León, \\
UANL. México & 12 \\
\hline Uniformed Services University of the \\
Health Sciences, \\
Maryland
\end{tabular}

\begin{tabular}{|c|c|}
\hline Drexel University & 3 \\
\hline Universidad de Vic & 3 \\
\hline Universidad del Rosario, Bogóta & 4 \\
\hline Universidad del Norte, Colombia & 1 \\
\hline Universidad de Tarapacá, Chile & 3 \\
\hline Universidad de Valparaíso, Chile & 1 \\
\hline $\begin{array}{c}\text { Universidad Nacional "Federico } \\
\text { Villarreal", Lima }\end{array}$ & 1 \\
\hline Universidad del País Vasco & 2 \\
\hline University of Calgary & 2 \\
\hline University of Leicester & 1 \\
\hline University of South Florida & 3 \\
\hline Universidad de los Andes, Bogotá & 3 \\
\hline Universidad Pública de Navarra & 2 \\
\hline $\begin{array}{c}\text { Centro Universitario Santa Ana } \\
\text { (Almendralejo) }\end{array}$ & 2 \\
\hline University of Maine & 1 \\
\hline Universidad Católica del Uruguay & 1 \\
\hline Universidad de Alcalá. Madrid & 1 \\
\hline University of Toronto & 1 \\
\hline McMaster University & 5 \\
\hline University of California San Francisco & 2 \\
\hline Universidad de los Pacientes & 2 \\
\hline Escuela Andaluza de Salud Pública & 6 \\
\hline Universidad de Sevilla & 2 \\
\hline Universidade de Coimbra, Portugal & 4 \\
\hline Universidad Veracruzana. México & 1 \\
\hline Total de firmas & 497 \\
\hline
\end{tabular}

Fuente: elaboración propia.

\section{Tabla 7. Producción por afiliación institucional de los autores: Hospitales/Centros médicos}

\begin{tabular}{|c|c|}
\hline $\begin{array}{c}\text { Producción por Hospitales/Centros } \\
\text { médicos }\end{array}$ & $\begin{array}{c}\text { Número } \\
\text { de firmas }\end{array}$ \\
\hline $\begin{array}{c}\text { ESAD 7. Servicio Madrileño de Salud. } \\
\text { Consejería de Sanidad. Comunidad de } \\
\text { Madrid }\end{array}$ & 2 \\
\hline $\begin{array}{c}\text { ESAD 2. Servicio Madrileño de Salud. } \\
\text { Consejería de Sanidad. Comunidad de } \\
\text { Madrid }\end{array}$ & 1 \\
\hline $\begin{array}{c}\text { ESAD 11. Servicio Madrileño de Salud. } \\
\text { Consejería de Sanidad. Comunidad de } \\
\text { Madrid }\end{array}$ & 1 \\
\hline $\begin{array}{c}\text { Hospital General Universitario Gregorio } \\
\text { Marañón de Madrid }\end{array}$ & 23 \\
\hline Hospital Clínico San Carlos. Madrid & 67 \\
\hline Hospital Universitario 12 de Octubre de \\
Madrid
\end{tabular}

\begin{tabular}{|c|c|}
\hline Hospital Universitario La Paz. Madrid & 81 \\
\hline Hospital Infantil Niño Jesús de Madrid & 15 \\
\hline Hospital Universitario La Fe. Valencia & 33 \\
\hline $\begin{array}{c}\text { Hospital Doctor Moliner de Valencia } \\
\text { Hospital Universitario Marqués de } \\
\text { Valdecilla }\end{array}$ & 1 \\
\hline $\begin{array}{c}\text { Hospital Ramón y Cajal. Madrid } \\
\text { Centro de Salud Mental de Huesca }\end{array}$ & 8 \\
\hline Hospital de Navarra & 19 \\
\hline $\begin{array}{c}\text { Centro de Salud de Mancha Real (Distrito } \\
\text { Sanitario de Jaén) }\end{array}$ & 1 \\
\hline Hospital Central de Defensa & 10 \\
\hline Anderson Cancer Center & 1 \\
\hline Hospital de Cabueñes. Gijón & 9 \\
\hline Hospital Donostia. San Sebastián & 3 \\
\hline
\end{tabular}




\begin{tabular}{|c|c|}
\hline $\begin{array}{l}\text { Hospital Universitario Sant Joan. Reus } \\
\text { (Tarragona) }\end{array}$ & 20 \\
\hline $\begin{array}{l}\text { Hospital Duran y Reynals. L'Hospitalet de } \\
\text { Llobregat }\end{array}$ & 21 \\
\hline Hospital de Terrassa. Barcelona & 1 \\
\hline Hospital do Meixoeiro, Vigo & 11 \\
\hline Hospital Puerta de Hierrro de Madrid & 1 \\
\hline $\begin{array}{l}\text { Complejo Hospitalario Universitario } \\
\text { Médico-Quirúrgico Ciudad de Jaén }\end{array}$ & 5 \\
\hline $\begin{array}{l}\text { Centro de Recuperación y Rehabilitación } \\
\text { de Levante }\end{array}$ & 1 \\
\hline $\begin{array}{l}\text { Hospital San Juan de Dios. Esplugues de } \\
\text { Llobregat, Barcelona }\end{array}$ & 2 \\
\hline Hospital General Yagüe. Burgos & 9 \\
\hline $\begin{array}{l}\text { Hospital Clínico Universitario de Santiago } \\
\text { de Compostela }\end{array}$ & 3 \\
\hline $\begin{array}{l}\text { Christie Hospital National Health Service } \\
\text { Trust. Manchester }\end{array}$ & 1 \\
\hline Fox Chase Cancer Center & 4 \\
\hline Hospital Josep Trueta. Girona & 3 \\
\hline Hospital General Universitario de Valencia & 24 \\
\hline Hospital Marina Alta. Denia. Alicante & 1 \\
\hline Hospital de Móstoles & 2 \\
\hline Hospital Virgen de la Paloma & 1 \\
\hline Hospital Universitario Udine. Italia & 2 \\
\hline Hospital Clínico Universitario Valencia & 12 \\
\hline Hospital General Universitario de Elche & 2 \\
\hline Hospital Carlos III. Madrid & 6 \\
\hline $\begin{array}{c}\text { Hospital de la Santa Creu i Sant Pau. } \\
\text { Barcelona }\end{array}$ & 7 \\
\hline $\begin{array}{c}\text { Hospital Universitario Miguel Servet. } \\
\text { Zaragoza }\end{array}$ & 2 \\
\hline $\begin{array}{c}\text { Hospital Universitario Reina Sofía. } \\
\text { Córdoba }\end{array}$ & 7 \\
\hline Hospital Universitario de Basurto. Bilbao & 7 \\
\hline Centro Agarimo. Pontevedra & 1 \\
\hline $\begin{array}{l}\text { Hospital Oncológico "Luis Razetti". } \\
\text { Caracas, Venezuela }\end{array}$ & 2 \\
\hline $\begin{array}{l}\text { Hospital Universitario Virgen de las } \\
\text { Nieves. Granada }\end{array}$ & 14 \\
\hline Hospital Universitario de Cruces. Vizcaya & 4 \\
\hline Hospital Español de México & 2 \\
\hline Hospital Médica Sur & 1 \\
\hline Hospital Príncipe de Asturias. Madrid & 8 \\
\hline $\begin{array}{c}\text { Centre Sociosanitari El Carme (Badalona } \\
\text { Serveis Assistencials, BSA) }\end{array}$ & 1 \\
\hline $\begin{array}{l}\text { Centro de Salud de Bidebieta-La Paz de } \\
\text { San Sebastián }\end{array}$ & 1 \\
\hline Centro de Salud Alza San Sebastián & 1 \\
\hline Hospital Pare Jofre.Valencia & 6 \\
\hline Hospital Virgen de la Poveda (SERMAS) & 3 \\
\hline $\begin{array}{c}\text { Servicio de Salud del Principado de } \\
\text { Asturias (SESPA) }\end{array}$ & 1 \\
\hline
\end{tabular}

\begin{tabular}{|c|c|}
\hline $\begin{array}{l}\text { Hospital Oncológico Marie Curie, Buenos } \\
\text { Aires }\end{array}$ & 1 \\
\hline $\begin{array}{l}\text { Hospital Clínico Universitario Lozano } \\
\text { Blesa, Zaragoza }\end{array}$ & 8 \\
\hline $\begin{array}{c}\text { Clínica Universidad Católica- Christus } \\
\text { Health }\end{array}$ & 1 \\
\hline $\begin{array}{c}\text { Hospital Universitario Son Espases. Palma } \\
\text { de Mallorca }\end{array}$ & 3 \\
\hline Hospital Universitari Vall d'Hebron & 7 \\
\hline $\begin{array}{l}\text { Centro de Investigación y Desarrollo en } \\
\text { Ciencias de la Salud, CIDICS }\end{array}$ & 3 \\
\hline $\begin{array}{l}\text { Hospital Universitario Dr. Peset de } \\
\text { Valencia }\end{array}$ & 1 \\
\hline Departamento Salud La Fe de Valencia & 1 \\
\hline Hospital Quirón Torrevieja & 4 \\
\hline $\begin{array}{l}\text { Hospital General de la Santísima Trinidad, } \\
\text { Salamanca }\end{array}$ & 1 \\
\hline Hospital Comarcal Sierrallana & 1 \\
\hline Hospital Mateu Orfila, Menorca & 2 \\
\hline Massachusetts General Hospital & 2 \\
\hline $\begin{array}{c}\text { Memorial Sloan-Kettering Cancer Center, } \\
\text { New York }\end{array}$ & 5 \\
\hline Hospital Parc Taulí de Sabadell & 2 \\
\hline Hospital Virgen de la Luz. Cuenca & 12 \\
\hline Hospital de San Pedro, Logroño & 3 \\
\hline Hospital General de la Rioja & 1 \\
\hline Hospital Santa Rita, Porto Alegre, Brasil & 1 \\
\hline City of Hope, Duarte & 4 \\
\hline Hospital San Pedro de Alcántara. Cáceres & 2 \\
\hline Tom Baker Cancer Centre & 2 \\
\hline $\begin{array}{l}\text { Hospital Centro de Cuidados Laguna. } \\
\text { Madrid }\end{array}$ & 3 \\
\hline Hospital Universitario La Princesa & 1 \\
\hline Hospital Universitario de Salamanca & 3 \\
\hline Hospital Río Carrión de Palencia & 1 \\
\hline $\begin{array}{c}\text { Unidad de Daño Cerebral Hermanas } \\
\text { Hospitalarias. Valencia }\end{array}$ & 12 \\
\hline $\begin{array}{l}\text { Hospital Sant Joan de Déu, Palma de } \\
\text { Mallorca }\end{array}$ & 1 \\
\hline $\begin{array}{c}\text { Hospital San Rafael de los Hermanos de } \\
\text { San Juan de Dios, Madrid }\end{array}$ & 1 \\
\hline $\begin{array}{l}\text { Centro de Psicología del Lenguaje y } \\
\text { Psicoterapia "ETHOS". Pamplona }\end{array}$ & 1 \\
\hline Hospital San Juan de Dios. Burgos & 3 \\
\hline $\begin{array}{l}\text { Centro de Rehabilitación } \\
\text { Neuropsicológica. Bogotá }\end{array}$ & 1 \\
\hline $\begin{array}{c}\text { Hospital de Pediatría Prof. J. P. Garrahan. } \\
\text { Ciudad de Buenos Aires }\end{array}$ & 1 \\
\hline $\begin{array}{c}\text { Tumor Biology Center at the University of } \\
\text { Freiburg }\end{array}$ & 3 \\
\hline Eastern Maine Medical Center & 1 \\
\hline $\begin{array}{c}\text { Hospital Psiquiátrico Universitario Institut } \\
\text { Pere Mata. Reus }\end{array}$ & 1 \\
\hline
\end{tabular}




\begin{tabular}{|c|c|}
\hline Complejo Asistencial de Segovia & 1 \\
\hline Atención Primaria del Área de Cuenca & 1 \\
\hline $\begin{array}{c}\text { Hospital de Día de Adultos. Parque } \\
\text { Sanitario Martí i Julià }\end{array}$ & 1 \\
\hline $\begin{array}{c}\text { Complejo Hospitalario Universitario de } \\
\text { Albacete }\end{array}$ & 1 \\
\hline Hospital Costa del Sol & 1 \\
\hline Hospital Universitario Puerta del Mar & 2 \\
\hline Hospital Universitario Virgen Macarena & 1 \\
\hline Hospital Universitario Virgen del Rocío & 2 \\
\hline Hospital Juan Ramón Jiménez. Huelva & 2 \\
\hline Hospital Regional de Málaga & 1 \\
\hline Hospital Infanta Elena & 1 \\
\hline Hospital de Torrecárdenas & 1 \\
\hline Distrito de Atención Primaria Málaga & 1 \\
\hline Distrito de Atención Primaria Sevilla & 1 \\
\hline Hospital de Mataró & 1 \\
\hline Hospital Universitario de Bellvitge & 1 \\
\hline Centro Asistencial San Camilo, Madrid & 1 \\
\hline
\end{tabular}

\begin{tabular}{|c|c|}
\hline $\begin{array}{l}\text { Hospital Universitario Madrid } \\
\text { Montepríncipe }\end{array}$ & 1 \\
\hline $\begin{array}{l}\text { Hospital Universitario Virgen de la } \\
\text { Arrixaca. Murcia }\end{array}$ & 2 \\
\hline Bellevue Hospital Center & 1 \\
\hline Guy's Hospital (London) & 1 \\
\hline $\begin{array}{l}\text { C. Sociosanitario N.S. del Carmen, } \\
\text { Valencia }\end{array}$ & 6 \\
\hline $\begin{array}{l}\text { Hospital-Residència Sant Camil. Sant Pere } \\
\text { de Ribes, Barcelona }\end{array}$ & 1 \\
\hline Hospital Clínic de Barcelona & 3 \\
\hline Centro de Salud Tórtola, Valladolid & 1 \\
\hline Centro de Salud La Victoria, Valladolid & 1 \\
\hline $\begin{array}{c}\text { Hospital Universitario del Río Hortega de } \\
\text { Valladolid }\end{array}$ & 1 \\
\hline Hospital de Sagunto & 1 \\
\hline Hospital Verge de la Cinta Tortosa & 1 \\
\hline Hospital General No. 45. México & 1 \\
\hline Total de firmas & 665 \\
\hline
\end{tabular}

Fuente: elaboración propia.

\section{Tabla 8. Producción por afiliación institucional de los autores: Asociaciones/Institutos profesionales}

$\left.\begin{array}{|c|c|}\hline \begin{array}{c}\text { Producción por Asociaciones/Institutos } \\ \text { profesionales }\end{array} & \begin{array}{c}\text { Número } \\ \text { de firmas }\end{array} \\ \hline \text { The Danish Cancer Society } & 1 \\ \hline \text { AECC-Cataluña contra el Cáncer } & 3 \\ \hline \text { AECC. Junta provincial Valencia } & 12 \\ \hline \text { AECC. Junta provincial de Granada } & 1 \\ \hline \text { AECC. Junta provincial de Cáceres } & 1 \\ \hline \text { AECC Junta Provincial La Rioja } & 1 \\ \hline \text { AECC. Junta provincial de Almería } & 3 \\ \hline \text { AECC. Cuenca } & 3 \\ \hline \text { AECC. Jaén } & 1 \\ \hline \text { AECC. Tenerife } & 1 \\ \hline \text { AECC. Barcelona } & 5 \\ \hline \text { Oficina de Coordinación Oncológica de la } \\ \text { Comunidad Autónoma de Madrid }\end{array}\right)$

\begin{tabular}{|c|c|}
\hline $\begin{array}{l}\text { Instituto Clínico de Neurociencias. } \\
\text { Barcelona }\end{array}$ & 4 \\
\hline $\begin{array}{c}\text { Institut d'Assisténcia Sanitaria, Unitat de } \\
\text { TMS. SALT (Girona) }\end{array}$ & 1 \\
\hline Corporación Sanitaria Clinic. Barcelona & 2 \\
\hline $\begin{array}{c}\text { Corporación Sanitaria Parc Taulí. Sabadell, } \\
\text { Barcelona }\end{array}$ & 2 \\
\hline $\begin{array}{l}\text { Fundación IMOR (Institut Médic Onco- } \\
\text { Radioterápia). Barcelona }\end{array}$ & 2 \\
\hline $\begin{array}{c}\text { Fundació Gastroenterología Dr. Francisco } \\
\text { Vilardell. Barcelona }\end{array}$ & 2 \\
\hline $\begin{array}{c}\text { Fundació per a la Investigació i Prevenció } \\
\text { del Càncer (FUNCA). Reus }\end{array}$ & 3 \\
\hline $\begin{array}{c}\text { Fundación Valenciana para el Estudio y } \\
\text { Tratamiento del Dolor }\end{array}$ & 1 \\
\hline $\begin{array}{c}\text { Centro de Promoción de Hábitos } \\
\text { Saludables. Ayuntamiento de Madrid }\end{array}$ & 1 \\
\hline $\begin{array}{l}\text { Instituto de Cancerología. Unidad de } \\
\text { Psicooncología. Medellín (Colombia) }\end{array}$ & 1 \\
\hline Asociación AMAC-GEMA. Zaragoza & 2 \\
\hline Instituto de Salud Carlos III & 14 \\
\hline Asociación Española de Derecho Sanitario & 1 \\
\hline $\begin{array}{l}\text { Federación Española de Cáncer de Mama } \\
\text { (FECMA) }\end{array}$ & 1 \\
\hline Bufete de Lorenzo Abogados & 1 \\
\hline
\end{tabular}




\begin{tabular}{|c|c|}
\hline Sociedad Española de Psicooncología & 1 \\
\hline Instituto de Psicología Antäe. Madrid & 2 \\
\hline $\begin{array}{c}\text { Instituto Nacional de Cancerología de } \\
\text { México }\end{array}$ & 46 \\
\hline $\begin{array}{l}\text { Instituto de Salud Pública. Comunidad de } \\
\text { Madrid }\end{array}$ & 2 \\
\hline $\begin{array}{c}\text { Junta de Andalucía. Consejería de Salud. } \\
\text { Servicio Andaluz de Salud }\end{array}$ & 2 \\
\hline $\begin{array}{c}\text { Instituto de Cancerología. Clínica Las } \\
\text { Américas }\end{array}$ & 1 \\
\hline $\begin{array}{l}\text { Asociación Latinoamericana de Cuidados } \\
\text { Paliativos }\end{array}$ & 1 \\
\hline Audiencia Provincial de Valencia & 1 \\
\hline I.E.S Pedreguer (Alicante) & 1 \\
\hline $\begin{array}{l}\text { Fundació Lliga Catalana d'Ajuda } \\
\text { Oncològica } \\
\end{array}$ & 1 \\
\hline $\begin{array}{c}\text { Organización Iberoamericana de la } \\
\text { Seguridad Social (OISS) }\end{array}$ & 1 \\
\hline $\begin{array}{l}\text { Instituto Maimónides de Investigación } \\
\text { Biomédica de Córdoba (IMIBIC) }\end{array}$ & 3 \\
\hline Instituto Nacional de Estadísticas de Chile & 1 \\
\hline $\begin{array}{c}\text { Instituto Investigaciones Sanitarias Pere } \\
\text { Virgili }\end{array}$ & 3 \\
\hline Instituto Nacional de Pediatría. México & 5 \\
\hline $\begin{array}{c}\text { Fundació Oncolliga, Lliga Catalana } \\
\text { d'Ajuda Oncològica }\end{array}$ & 1 \\
\hline $\begin{array}{c}\text { Instituto Nacional de Psiquiatría Ramón de } \\
\text { la Fuente Muñiz. México }\end{array}$ & 3 \\
\hline $\begin{array}{c}\text { Instituto de Medicina Predictiva i } \\
\text { Personalizada del Cáncer (IMPPC), } \\
\text { Badalona }\end{array}$ & 1 \\
\hline FEFOC, Barcelona & 3 \\
\hline Fundación TEDECA & 4 \\
\hline $\begin{array}{l}\text { Instituto Oncológico Henry Moore. } \\
\text { Buenos Aires }\end{array}$ & 8 \\
\hline Instituto William Osler. Buenos Aires & 9 \\
\hline $\begin{array}{l}\text { Unidad de investigación en Psiquiatría de } \\
\text { Cantabria }\end{array}$ & 2 \\
\hline Fundación Jiménez Díaz & 2 \\
\hline $\begin{array}{l}\text { Instituto Mexicano de Seguro Social, } \\
\text { Querétaro, México }\end{array}$ & 5 \\
\hline $\begin{array}{l}\text { Agencia Laín Entralgo. Consejería de } \\
\text { Sanidad, Comunidad de Madrid }\end{array}$ & 8 \\
\hline $\begin{array}{c}\text { Coordinación Regional de Cuidados } \\
\text { Paliativos. Consejería de Sanidad, } \\
\text { Comunidad de Madrid }\end{array}$ & 2 \\
\hline $\begin{array}{c}\text { Instituto Nacional de Ciencias Médicas y } \\
\text { Nutrición "Salvador Zubirán" }\end{array}$ & 1 \\
\hline Institut Oncològic del Vallès & 6 \\
\hline Consorci Sanitari de Terrassa & 8 \\
\hline $\begin{array}{c}\text { INSPIRA. Desarrollo Personal y } \\
\text { Aprendizaje Organizativo }\end{array}$ & 1 \\
\hline
\end{tabular}

\begin{tabular}{|c|c|}
\hline $\begin{array}{c}\text { Fundación "la Caixa". Área de Integración } \\
\text { Social. Barcelona }\end{array}$ & 2 \\
\hline Fundación Grupo IMO & 2 \\
\hline Fundación Rioja Salud & 1 \\
\hline Instituto Politécnico Nacional, México & 1 \\
\hline Fundación Aladina & 1 \\
\hline $\begin{array}{l}\text { Unidad de investigación en Psiquiatría de } \\
\text { Cantabria }\end{array}$ & 3 \\
\hline $\begin{array}{l}\text { Centro de Investigación en Alimentación y } \\
\text { Desarrollo, A.C. Hermosillo, México }\end{array}$ & 1 \\
\hline $\begin{array}{l}\text { Unidad de Comunicación Médica. } \\
\text { Osakidetza }\end{array}$ & 1 \\
\hline Leicestershire Partnership Trust & 1 \\
\hline Agència de Salut Pública de Barcelona & 5 \\
\hline CIBER de Epidemiología y Salud Pública & 7 \\
\hline $\begin{array}{l}\text { Institut d'Investigació Biomèdica Sant Pau } \\
\text { (IIB Sant Pau), Barcelona }\end{array}$ & 3 \\
\hline $\begin{array}{c}\text { Institut Universitari d'Investigació en } \\
\text { Atenció Primaria Jordi Gol (IDIAP Jordi } \\
\text { Gol), Barcelona }\end{array}$ & 1 \\
\hline Generalidad Valenciana & 1 \\
\hline Fundación Santafé de Bogotá & 1 \\
\hline Obra Social "La Caixa. Madrid & 2 \\
\hline Asociación Navarra de Laringectomizados & 1 \\
\hline GASMEDI 2000 S.A.U. & 1 \\
\hline Asociación Oncológica Extremeña (AOEx) & 8 \\
\hline $\begin{array}{c}\text { Fundación Canaria de Investigación y } \\
\text { Salud (FUNCIS) }\end{array}$ & 4 \\
\hline Fundación Josep Laporte & 2 \\
\hline Fòrum Català de Pacients & 1 \\
\hline Plan Integral de Oncología de Andalucía & 1 \\
\hline Comité de Bioética de Cataluña & 1 \\
\hline Consorci Sanitari del Maresme, Mataró & 1 \\
\hline $\begin{array}{c}\text { Egarsat, Mutua de Accidentes de Trabajo } \\
\text { de la Seguridad Social, núm. } 76\end{array}$ & 1 \\
\hline $\begin{array}{c}\text { Servicios Sociales Comunitarios. } \\
\text { Ayuntamiento de Vícar }\end{array}$ & 1 \\
\hline Institut Català d'Oncologia-Girona & 2 \\
\hline Atención Psicosocial de "La Caixa" & 1 \\
\hline $\begin{array}{l}\text { Servicio Especializado de Atención a la } \\
\text { Familia e Infancia (SEAFI), Quart de Poblet }\end{array}$ & 1 \\
\hline Consorci Sanitari del Garraf & 1 \\
\hline Fundación Pequeño Deseo & 2 \\
\hline $\begin{array}{c}\text { CONACYT-UNAM. Instituto Nacional de } \\
\text { Psiquiatría }\end{array}$ & 2 \\
\hline $\begin{array}{c}\text { Instituto para el Fortalecimiento de } \\
\text { Capacidades en Salud (FOCUS Salud } \\
\text { México) }\end{array}$ & 2 \\
\hline Total de firmas & 371 \\
\hline
\end{tabular}

Fuente: elaboración propia. 


\section{Temática de las contribuciones}

Las palabras clave sirven para designar el contenido del trabajo. Se han contabilizado un total de 1582 palabras clave, muchas de las cuales son sinónimas, destacando el problema de la no normalización de los conceptos.

Las materias más frecuentes han sido «cáncer», «cáncer de mama», «calidad de vida», «cuidados paliativos», «depresión», etc. En la tabla 9 se han reflejado las palabras clave que presentan una frecuencia de al menos siete casos, pero ello supone dejar fuera de este cómputo 1555palabras clave (el 98\%). La palabra clave «cáncer» se posiciona en primer lugar (4,7\%).
- El ritmo productivo de las contribuciones es regular, concentrándose el mayor número de trabajos en el año 2004.

- El grupo dirigente en cuanto a producción lo componen en su mayor parte profesores de la Facultad de Psicología de la Universidad Complutense, entidad editora de la revista. En concordancia con lo anterior, la institución más productiva es la Universidad Complutense.

- Sería conveniente una mayor normalización en el empleo de las palabras clave por parte de los autores, proponiendo el uso del MeSH (Tesauro de MEDLINE) o de la Cla-

\section{Tabla 9. Palabras clave con mayor frecuencia}

\begin{tabular}{|c|c|c|c|}
\hline Palabras clave & Número & Palabras clave & Número \\
\hline Afrontamiento & 12 & Duelo & 7 \\
\hline Ansiedad & 34 & Eficacia & 7 \\
\hline Astenia & 8 & Evaluación & 10 \\
\hline Calidad de vida & 58 & Imagen corporal & 14 \\
\hline Cáncer & 75 & Intervención psicológica & 12 \\
\hline Cáncer de mama & 58 & Malestar emocional & 18 \\
\hline Cáncer de pulmón & 8 & Oncología & 16 \\
\hline Cáncer hereditario & 7 & Psicooncología & 19 \\
\hline Cáncer infantil & 7 & Quimioterapia & 12 \\
\hline Comunicación & 15 & Radioterapia & 7 \\
\hline Consejo genético & 17 & Toma de decisiones & 8 \\
\hline Cuidados paliativos & 37 & Tratamiento & 8 \\
\hline Depresión & 39 & Tratamiento psicológico & \\
\hline Dolor & 8 & & \\
\hline
\end{tabular}

Fuente: elaboración propia.

\section{CONCLUSIONES}

El análisis bibliométrico efectuado nos permite extraer las siguientes conclusiones:

- La revista se ha consolidado tras más de una década de existencia con una producción media que se sitúa en torno a los 29 artículos. sificación Internacional de Enfermedades CIE-10.

- A pesar del predominio de autores de género femenino $(64,26 \%)$ la mayoría de los autores más productivos son de género masculino. En términos de producción de trabajos-fuente el autor Juan Antonio Cruzado (de la Universidad Com- 
plutense de Madrid) se posiciona en primer lugar.

- La mayoría de los autores firman con más de una filiación.

- La procedencia geográfica internacional de los autores de los artículos se concentra en México. Desde el primer momento la revista ha acogido la colaboración de autores extranjeros en el Consejo de Redacción que, vinculados a universidades e instituciones o a título personal, han contribuido significativamente a la apertura y proyección internacional de la revista.

- En España, la Comunidad de Madrid se configura como la más productiva.

- Sería conveniente una mayor unificación en la firma de los artículos. Hay autores que emplean distintas variantes como el uso de iniciales (Ej. Juan A. Cruzado o Juan Antonio Cruzado).

- El análisis de materias indica los siguientes intereses por parte de los autores: en primer lugar, aparecen los temas médicos asociados al cáncer (tipos de cáncer, tratamientos y otras cuestiones profesionales). En segundo lugar, encontramos la categoría que recoge trabajos relacionados con aspectos psicológicos asociados a la enfermedad. En tercer lugar, trabajos sobre cuidados y comunicación con el paciente, destacando los artículos que tratan cuestiones de relación médico-paciente.

\section{REFERENCIAS BIBLIOGRÁFICAS}

1. Burgos Bordonau, E, Palacios Gómez JL. Estudio bibliométrico y de calidad de la revista Cuadernos de Trabajo Social (1987-2008). Cuadernos de Trabajo Social 2009; 22:83-108. Doi: 10.5209

2. Instituto Nacional de Estadística (INE). (2016). En Linea. [Acceso 10 de abril de 2016]. Disponible en: http://www.ine. es/jaxi/menu.do?type $=$ pcaxis \&path $=/$ t15/ p417/\&file=inebase 